\title{
Robust Bayesian nonlinear mixed effects modeling of time to positivity in tuberculosis trials
}

\author{
Divan Aristo Burger ${ }^{1} \mid$ Robert Schall ${ }^{2,3}$ | Ding-Geng Chen ${ }^{1}$ \\ ${ }^{1}$ Department of Statistics, University of Pretoria, Pretoria, South Africa \\ ${ }^{2}$ Department of Mathematical Statistics and Actuarial Science, University of the Free State, Bloemfontein, South Africa \\ ${ }^{3}$ IQVIA, Biostatistics, Bloemfontein, South Africa
}

KEY WORDS: Bayesian, heavy tailed, mixed effects, nonlinear, time to positivity

Address correspondence to Divan Aristo Burger, Department of Statistics, University of Pretoria, Pretoria 0028, South Africa; E-mail: divanaburger@gmail.com 


\section{ABSTRACT}

Early Phase 2 tuberculosis (TB) trials are conducted to characterize the early bactericidal activity (EBA) of anti-TB drugs. The EBA of anti-TB drugs has conventionally been calculated as the rate of decline in colony forming unit (CFU) count during the first 14 days of treatment. The measurement of CFU count, however, is expensive and prone to contamination. Alternatively to CFU count, time to positivity (TTP), which is a potential biomarker for long-term efficacy of anti-TB drugs, can be used to characterize EBA. The current Bayesian nonlinear mixed effects (NLME) regression model for TTP data, however, lacks robustness to gross outliers that often are present in the data. The conventional way of handling such outliers involves their identification by visual inspection and subsequent exclusion from the analysis. However, this process can be questioned because of its subjective nature. For this reason, we fitted robust versions of the Bayesian NLME regression model to a wide range of TTP datasets. The performance of the explored models was assessed through model comparison statistics and a simulation study. We conclude that fitting a robust model to TTP data obviates the need for explicit identification and subsequent "deletion" of outliers, but ensures that gross outliers exert no undue influence on model fits. We recommend that the current practice of fitting conventional normal theory models be abandoned in favor of fitting robust models to TTP data. 


\section{INTRODUCTION}

\subsection{Tuberculosis surrogate endpoints}

The primary endpoints of pivotal Phase 3 tuberculosis (TB) trials are the proportion of patients with positive sputum culture after 6 months of treatment, and the proportion of patients experiencing relapse within a two-year follow-up period. ${ }^{[1,2]}$ Such trials, therefore, are lengthy and expensive. ${ }^{[1]}$ In contrast, the use of an appropriate surrogate marker for the effectiveness of TB treatments can shorten the duration of anti-TB drug development, and might predict efficacy early during a TB drug's development phase. ${ }^{[3]}$

Biomarkers should be able to predict the long-term outcome of the disease, such as potential relapses. Sputum culture status ("positive" or "negative") after two months of treatment is considered a potential surrogate marker for the aforementioned primary efficacy TB endpoints. ${ }^{[4]}$ Limitations of this surrogate marker, however, are that large sample sizes are required, and its lack of association with relapse within individual patients. ${ }^{[5]}$ Two alternatives to the two-month culture status surrogate marker are the bactericidal activity as characterized by the rate of decline in colony forming unit (CFU) count over 56 days of treatment, and bactericidal activity as characterized by the rate of increase in time to positivity (TTP) over 56 days of treatment. ${ }^{[5,6]}$

TTP is the time it takes for a given sputum sample to yield a positive Mycobacteria Growth Indicator Tube (MGIT) culture. If no positive MGIT culture is reported after a specified maximum number of hours, the sputum sample status is reported as "no mycobacterial growth". ${ }^{[7]}$ The TTP endpoint is considered more sensitive than CFU count in the evaluation

of TB treatments. ${ }^{[8]}$ Moreover, the collection of CFU counts is prone to contamination, and involves a time consuming and expensive counting process. ${ }^{[9]}$ Early bactericidal activity (EBA) calculated from TTP data has therefore recently become the preferred surrogate marker (although TTP measurements may also be prone to contamination). ${ }^{[8]}$ 
Recent literature pertaining to the statistical modeling of TTP data includes the following: Kassir ${ }^{[18]}$ proposed a two-part longitudinal model for the change in TTP over time, and probability of no mycobacterial growth. Chigutsa et al. ${ }^{[19]}$ introduced a semimechanistic model for TTP data using a time-to-event modeling approach. Svensson and Karlsson ${ }^{[2]}$ suggested an approach that simultaneously (i) incorporates a latent variable representing the bacterial load in the human body, (ii) models the probability of no mycobacterial growth, and (iii) includes a survival analysis counterpart for TTP data.

\subsection{Bactericidal activity trials of tuberculosis drugs}

In EBA trials of TB drugs patients are randomized to one of several treatments to be studied (say, three to five test treatments and a control treatment representing best available care). At baseline, and during the treatment period serial sputum samples are taken from the patients, typically over 14 days. TTP is determined for each sputum sample, so that for each patient a TTP versus time profile is obtained.

The EBA parameter calculated from a TTP versus time profile has been expressed as the daily percentage change in TTP over a certain time interval as follows ${ }^{[10]}$ :

$$
\operatorname{EBA}\left(t_{1}-t_{2}\right)=100\left(10 \frac{\hat{f}\left(t_{2}\right)-\hat{f}\left(t_{1}\right)}{t_{2}-t_{1}}-1\right)
$$

where $f(t)$ is a suitable regression function for TTP, on the logarithmic scale to the base of 10 , against time. The associated fitted values at Day $t_{1}$ and Day $t_{2}$ are respectively $\hat{f}\left(t_{1}\right)$ and $\hat{f}\left(t_{2}\right)$.

\subsection{Need for robust models for time to positivity}

Burger and Schall[ ${ }^{[11]}$ proposed a biphasic Bayesian nonlinear mixed effects (NLME) regression model for $\log _{10}$ (CFU) count which is more flexible than previously fitted regression models. This Bayesian NLME regression model has also successfully been fitted to TTP datasets of recently published EBA trials. ${ }^{[3,6,10]}$ The regression model fitted to these TTP datasets, however, 
assumed normally distributed random coefficients and residuals.

Investigation of a large number of TTP datasets showed that most individual TTP versus time profiles follow the model very closely, so that the model residuals are small in the majority of such profiles (see Section 3). Occasionally, however, data profiles contain gross outliers that severely deviate both from the pattern set by the majority of data profiles, and from the pattern set by the majority of data points within the profile in question (therefore suggesting that the distribution of TTP data may be heavy tailed and skewed). Many of these deviations are so large that they are biologically highly implausible, or even not possible. As an alternative to the specification of the conventional normal distribution, the Student $t$, skew Student $t$, slash and contaminated normal distribution may provide more robust fits for such heavy tailed data. ${ }^{[12-14]}$

In past EBA studies (e.g. Diacon et al. ${ }^{[15]}$ ) outliers in TTP data were manually identified during blind data review meetings, on a case-by-case basis, and excluded from the statistical analysis. Such processes, however, are not satisfactory and can be questioned because of the subjective decisions involved; regulatory guidelines generally advise against any removal of data from the analysis. ${ }^{[16]}$ On the other hand, it seems clearly unsatisfactory to fit a fairly intricate model to data points which medical experts consider biologically implausible.

In this paper we propose the fit of robust models to TTP data. Doing so both obviates the need for "deletion" of outliers (or even their explicit identification), and ensures that gross outliers exert no undue influence on the model fit. Specifically, the robust regression models of Burger and Schall[17] are applied to TTP data, in order to accommodate outliers and potential skewness in the distribution of the data. The empirical performance of the proposed methodology is investigated in an extensive range of datasets.

\subsection{Outline of present paper}

Section 2 briefly presents the NLME regression models introduced by Burger and Schall ${ }^{[17]}$ for $\log _{10}(\mathrm{CFU})$ count, and formulates them in terms of TTP data. In Section 3 the models presented in Section 2 are fitted to various TTP datasets of recently published clinical trials: 
In particular, the regression models are fitted to six 14-day datasets (EBA; Phase 2a), and one 56-day dataset (extended bactericidal activity; Phase 2b). Section 4 presents a simulation study to compare the performance of the regression models. Section 5 discusses the findings of the paper.

\section{BAYESIAN MIXED EFFECTS REGRESSION MODELS}

The investigation of TTP data versus time profiles has suggested that $\log _{10}($ TTP) increases linearly or bilinearly during the first few weeks of treatment. ${ }^{[15]}$ The Bayesian NLME regression model proposed by Burger and Schall[ ${ }^{[17]}$, now formulated in terms of TTP data, is as follows:

$$
\log _{10}\left(y_{i j k}\right)=\alpha_{i j}+\beta_{1 i j} \cdot t_{i j k}+\beta_{2 i j} \cdot \gamma_{i j} \cdot \log \left(\frac{e^{\frac{t_{i j k}-\kappa_{i j}}{\gamma_{i j}}}+e^{-\frac{t_{i j k}-\kappa_{i j}}{\gamma_{i j}}}}{e^{\frac{\kappa_{i j}}{\gamma_{i j}}}+e^{-\frac{\kappa_{i j}}{\gamma_{i j}}}}\right)+\varepsilon_{i j k}
$$

where $y_{i j k}$ is the TTP measurement for patient $i=1, \ldots, N_{j}$ in treatment group $j=1, \ldots, J$ at timepoint $k=1, \ldots, T_{i j}$, and $t_{i j k} \geq 0$ is the corresponding measurement time. Here, $\alpha_{i j}$ denote the random intercepts, $\beta_{1 i j}$, $\beta_{2 i j}$ the two random slopes (governing the rate of change over time), $\kappa_{i j}$ the inflection point (at which transition from one slope to another occurs), $\gamma_{i j}$ the "smoothness" parameters (governing the "speed" of transition), and $\varepsilon_{i j k}$ are the residuals.

The terms $\alpha_{i j}, \beta_{1 i j}, \beta_{2 i j}, \kappa_{i j}$ and $\gamma_{i j}$ are the sums of fixed effects and associated random coefficients, namely:

$$
\boldsymbol{\mu}_{i j}=\left[\begin{array}{l}
\alpha_{i j} \\
\beta_{1 i j} \\
\beta_{2 i j}
\end{array}\right]=\left[\begin{array}{l}
\alpha_{j} \\
\beta_{1 j} \\
\beta_{2 j}
\end{array}\right]+\left[\begin{array}{l}
u_{0 i j} \\
u_{1 i j} \\
u_{2 i j}
\end{array}\right]=\boldsymbol{\mu}_{j}+\left[\begin{array}{l}
u_{0 i j} \\
u_{1 i j} \\
u_{2 i j}
\end{array}\right]
$$

and

$$
\left[\begin{array}{c}
\kappa_{i j} \\
\gamma_{i j}
\end{array}\right]=\left[\begin{array}{l}
\kappa_{j} \\
\gamma_{j}
\end{array}\right]+\left[\begin{array}{l}
u_{3 i j} \\
u_{4 i j}
\end{array}\right]
$$


where $\boldsymbol{\mu}_{i j}$ and $\boldsymbol{\mu}_{j}$ are respectively the vectors of random and mean intercepts and slopes, and $\left(\kappa_{i j}, \gamma_{i j}\right)^{\prime}$ and $\left(\kappa_{j}, \gamma_{j}\right)^{\prime}$ are respectively the vectors of random and mean nodes and smoothness parameters.

Table 1 lists the various specifications of the Bayesian mixed effects regression model in Equation (1). ${ }^{[17]}$ In particular, the Student $t$ distribution is specified for the residuals and random coefficients, so as to yield a robust analysis of potentially skewed and heavy tailed TTP data. Detail on the corresponding distributions specified for residuals and random coefficients, including the corresponding noninformative prior distributions, is provided in the original paper, and summarized in the appendix of the present paper.

\section{APPLICATION}

\subsection{Computational issues}

The regression models were fitted using OpenBUGS. ${ }^{[20]}$ Posterior samples were monitored using iteration and autocorrelation plots, ${ }^{[21]}$ and Brooks-Gelman-Rubin statistics of parallel chains. ${ }^{[22]}$ Model discrimination statistics, namely the deviance information criterion (DIC) statistic and compound Laplace-Metropolis marginal likelihood (CLMML), were calculated in SAS ${ }^{\circledR}$ and the R project. The multidimensional integration library "R2Cuba" of the R project was used to approximate the Laplace integrals. ${ }^{[23]}$ It should be noted that DIC statistics compare the regression models conditional on the random effects, whereas CLMMLs compare models on a marginal basis.

\subsection{Early bactericidal activity}

\subsubsection{Datasets}

The regression models listed in Table 1 were fitted to a wide range of 14-day EBA datasets, namely data from the following six clinical trials (Phase 2a): CL001 ${ }^{[24]}: 5$ treatments, 68 pa- 
tients; CL007 ${ }^{[25]}$ : 5 treatments, 69 patients; CL010 ${ }^{[26]}: 5$ treatments, 69 patients; NC001 ${ }^{[15]}$ :

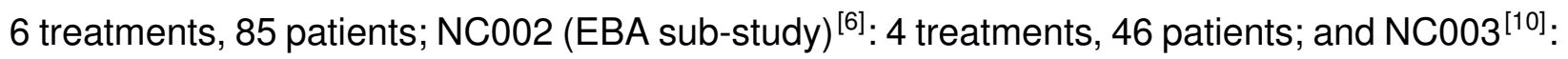
7 treatments, 101 patients (total: 6 datasets, 32 treatments groups, 438 patients). Results from the regression models, fitted jointly to the data of all patients (per trial), are presented below.

As mentioned above, TTP values might be reported as "no mycobacterial growth". The manufacturer's recommended incubation time before reporting a result as "no mycobacterial growth" is 42 days (or 1008 hours). ${ }^{[15]}$ Thus the largest possible numeric TTP value that can be observed is 1008 hours. In the context of the above model, when regressing $\log _{10}$ (TTP) against time, a "no mycobacterial growth" TTP value on the logarithmic scale to the base of 10 is specified as a right censored value of $\log _{10}(1008)$.

\subsubsection{Illustration of robustness of fits}

Plots of observed $\log _{10}(T T P)$ versus time profiles, together with fits of regression models $R_{N} C_{N}$ and $\mathrm{R}_{S T} \mathrm{C}_{\mathrm{T}}$, are included in Figure 1 and Figure 2 for eight patients in the NC003 trial (thus comparing fits of the most general (robust) regression model versus its non-robust counterpart). Figure 1 shows four typical profiles where the data points closely match the fitted curve; in fact, the $\log _{10}(\mathrm{TTP})$ versus time profiles of the majority of patients have this property. In contrast, Figure 2 shows four examples of profiles that contain gross outliers; here regression model $\mathrm{R}_{\mathrm{ST}} \mathrm{C}_{\mathrm{T}}$ provides robust fits of the regression curve, with little weight given to the various clinically implausible (or even impossible) TTP values.

For all treatment groups and all example datasets (that is, 32 treatment groups in total), we calculated Bayesian point and interval estimates for the primary efficacy parameter, mean $\operatorname{EBA}_{\mathrm{TTP}_{j}}(0-14)$. (We reported the mean and $2.5^{\text {th }} \& 97.5^{\text {th }}$ percentile of posterior distributions to respectively serve as Bayesian point and interval estimates.) Figure 3 presents the Bland-Altman plot of the posterior estimates of mean $\operatorname{EBA}_{T T P P_{j}}(0-14)$ for regression models $R_{N} C_{N}$ and $R_{S T} C_{T}$. The Bland-Altman plot shows differences between $R_{N} C_{N}$ and $R_{S T} C_{T}$ in the posterior estimates of mean $\operatorname{EBA}_{T_{T P}}(0-14)$ ranging from -0.363 to 0.174 (hence minor 
differences). For the robust regression models $R_{T} C_{N}, R_{S T} C_{N}$ and $R_{S T} C_{T}$, Figure 4 shows boxand-whisker plots of the ratio of the width of the $95 \%$ Bayesian credibility intervals (BCls) for mean $\operatorname{EBA}_{\text {TTP }_{j}}(0-14)$, relative to the non-robust model $\mathrm{R}_{\mathrm{N}} \mathrm{C}_{\mathrm{N}}$. A ratio of less than 1 indicates that the width of the $95 \% \mathrm{BCl}$ for the robust regression model in question is narrower than that of the non-robust model $\left(\mathrm{R}_{\mathrm{N}} \mathrm{C}_{\mathrm{N}}\right)$. The box-and-whisker plots show that the robust $95 \% \mathrm{BCls}$ are narrower than their non-robust counterpart in at least $75 \%$ of cases (about $5 \%$ narrower on average). The posterior estimates and corresponding $95 \% \mathrm{BCls}$ for mean $\mathrm{EBA}_{\mathrm{TTP}_{j}}(0-14)$ are similar for regression models $\mathrm{R}_{T} \mathrm{C}_{\mathrm{N}}, \mathrm{R}_{\mathrm{ST}} \mathrm{C}_{\mathrm{N}}$ and $\mathrm{R}_{\mathrm{ST}} \mathrm{C}_{\mathrm{T}}$ (data not shown).

\subsubsection{Distribution of residuals: Heavy tailed and skewed}

Again for all treatment groups and all example datasets (32 treatment groups in total), we calculated Bayesian estimates of the residual degrees of freedom and skewness parameters under the robust regression model $\left(\mathrm{R}_{\mathrm{ST}} \mathrm{C}_{\mathrm{T}}\right)$. Figure $5 \mathrm{a}$ and Figure $5 \mathrm{~b}$ respectively present those estimates, and provide evidence that the distribution of the model residuals often is heavy tailed and slightly skewed: Figure 5a shows that the estimates of degrees of freedom are below 10 in 25 out of 32 cases (suggesting a heavy tailed distribution of the residuals), and Figure 5b shows that the estimates of the skewness parameter are above 0 in 21 out of 32 cases (suggesting slight skewness to the right). In contrast, estimates for the degrees of freedom of the vectors of random intercepts and slopes are above 30 in 31 out of 32 cases (data not shown), which suggests that their distributions are not heavy tailed (that is, for all practical purposes follow normal distributions).

\subsubsection{Model comparison}

Model comparison statistics for the various Bayesian NLME regression models fitted are provided in Table 2 . The results presented in Table 2 suggest the following:

- Both model comparison statistics, namely DIC statistics and CLMMLs, select models with Student t distributed residuals $\left(\mathrm{R}_{T} \mathrm{C}_{N}\right)$ over the normal distribution $\left(\mathrm{R}_{N} \mathrm{C}_{N}\right)$ in all 
cases.

- The DIC statistics mainly select the skew Student $t$ distribution $\left(\mathrm{R}_{S T} \mathrm{C}_{\mathrm{N}}\right.$ and $\left.\mathrm{R}_{S T} \mathrm{C}_{\mathrm{T}}\right)$ for the residuals over the standard (symmetric) Student $t$ distribution $\left(\mathrm{R}_{T} \mathrm{C}_{\mathrm{N}}\right)$, whereas the CLMMLs generally select the standard Student t distribution $\left(\mathrm{R}_{T} \mathrm{C}_{\mathrm{N}}\right)$.

- Regarding random intercepts and slopes, the DIC statistics and CLMMLs in most cases indicate no distinct preference for the Student $t$ distribution $\left(\mathrm{R}_{\mathrm{ST}} \mathrm{C}_{\mathrm{T}}\right)$ over the normal distribution $\left(\mathrm{R}_{S T} \mathrm{C}_{N}\right)$ (the DIC and CLMML differences are negligible).

Overall, the model comparison statistics prefer regression model $R_{T} C_{N}$ in 8 out of 12 cases.

\subsubsection{Sensitivity analysis}

The regression model recommended in Section 3.2.4 introduces one parameter in addition to the regression model published by Dawson et al. (normal versus Student t). ${ }^{[6]}$ In a sensitivity analysis we therefore investigated the influence of the prior specification of the degrees of freedom on the inference about the EBA of treatments. In its basic form, regression model $\mathrm{R}_{\mathrm{T}} \mathrm{C}_{\mathrm{N}}$ specified uniform prior distributions for the degrees of freedom, namely $v_{j} \sim U(2,100)$ (see appendix). Alternatively, the $v_{j}$ were assigned weakly informative truncated gamma prior distributions, namely $v_{j} \sim G(2,0.01) I\left(v_{j} \geq 2\right)$, for which the density function is written as ${ }^{[27]}$ :

$$
f\left(v_{j}\right)= \begin{cases}\frac{2^{0.01}}{\Gamma(0.01)} e^{-2\left(v_{j}-2\right)}\left(v_{j}-2\right)^{0.01-1} & v_{j} \geq 2 \\ 0 & v_{j}<2\end{cases}
$$

It turns out that the inferences about the EBA of the 32 treatment groups are almost identical under both prior specifications of $v_{j}$ (data not shown). We therefore conclude that the EBA is not sensitive to the two types of prior specifications of $v_{j}$. 


\subsection{Extended bactericidal activity}

In addition to fitting the robust regression models to TTP data from EBA trials, we applied the robust methodology to TTP data from an extended bactericidal activity trial, NC002 (Phase 2b). ${ }^{6]}$ In this trial, drug-sensitive TB patients were randomized to receive 56-day combination therapy of either moxifloxacin, PA-824 (100 mg) and pyrazinamide (M-PA100-Z; 60 patients); or moxifloxacin, PA-824 (200 mg) and pyrazinamide (M-PA200-Z; 62 patients); or Rifafour (59 patients); whereas multi-drug resistant (MDR) TB patients were assigned to receive 56-day combination therapy of moxifloxacin, PA-824 (200 mg) and pyrazinamide (M-PA200-Z-MDR; 26 patients).

The posterior estimates and corresponding 95\% BCls for mean $\mathrm{EBA}_{\mathrm{TTP}_{j}}(0-56)$ from models $\mathrm{R}_{N} \mathrm{C}_{\mathrm{N}}$ and $\mathrm{R}_{\mathrm{T}} \mathrm{C}_{\mathrm{N}}$ are presented in Table 3. The posterior estimates and $95 \% \mathrm{BCls}$ of mean $\log _{10}$ (TTP) are shown in Figure 6 by treatment group and day. The estimates from regression model $R_{T} C_{N}$ are larger than those of regression model $R_{N} C_{N}$, while the $95 \% B C l s$ for regression model $R_{T} C_{N}$ are narrower than those of regression model $R_{N} C_{N}$. The DIC statistic and CLMML for regression model $R_{N} C_{N}$ are respectively -863.60 and -27.48 , and those for regression model $\mathrm{R}_{\mathrm{T}} \mathrm{C}_{\mathrm{N}}$ are respectively -1899.00 and 267.03. Thus both the DIC statistic and CLMML favor the model with Student $t$ distributed residuals $\left(\mathrm{R}_{T} \mathrm{C}_{\mathrm{N}}\right)$ over those with normally distributed residuals $\left(R_{N} C_{N}\right)$.

\section{SIMULATION STUDY}

We conducted a simulation study to assess the consequences of fitting (i) conventional normal theory regression models to TTP data containing outliers, and (ii) robust regression models to TTP data containing no outliers. Datasets were simulated from regression models $R_{N} C_{N}$ and $\mathrm{R}_{\mathrm{T}} \mathrm{C}_{\mathrm{N}}$ where model parameters were chosen to mimic TTP data of treatments that exhibit low and moderate levels of bactericidal activity.

The slope parameters for the two treatments were chosen as $\beta_{11}=0.0115, \beta_{21}=-0.002$, 
$\beta_{12}=0.0398$ and $\beta_{22}=-0.002$, while the following parameter values were chosen for both treatments (where applicable) $(j=1,2): \alpha_{j}=2, \kappa_{j}=5, \gamma_{j}=1, v_{j}=2, \sigma_{\varepsilon_{j}}^{2}=0.12$ and

$$
\Omega_{\mu j}=\left[\begin{array}{ccc}
0.5 & 0.0001 & 0.0001 \\
0.0001 & 0.01 & -0.0001 \\
0.0001 & -0.0001 & 0.01
\end{array}\right]
$$

Here, the EBA of the two treatments is $\operatorname{EBA}_{T_{T P}}(0-14)=1.0989$ and $\operatorname{EBA}_{\mathrm{TTP}_{2}}(0-14)=4.0008$.

The accuracy and precision characteristics bias, standard error (SE), and root mean square error (RMSE) of the $\mathrm{EBA}_{\mathrm{TTP}_{j}}(0-14)$ estimates for the two treatments were calculated. The corresponding empirical coverage probability of the $95 \%$ BCls was also calculated.

Regression models $R_{N} C_{N}$ and $R_{T} C_{N}$ were fitted to 1500 simulated datasets (simulated from $R_{N} C_{N}$ and $R_{T} C_{N}$ ), each dataset consisting of 15 patient profiles per treatment. For simplicity (to reduce computational burden), the regression models fitted $\kappa_{i j}$ and $\gamma_{i j}$ as fixed effects (i.e. $\kappa_{i j}=\kappa_{j}$ and $\gamma_{i j}=\gamma_{j}$ ).

From Table 4, we observe that the bias of $\operatorname{EBA}_{\mathrm{TTP}_{j}}(0-14)$ estimates is small for both models (whether the data are simulated from $R_{N} C_{N}$ or $R_{T} C_{N}$ ). When the data are simulated from $R_{T} C_{N}$, the SE and RMSE suggest that the robust regression model $\left(R_{T} C_{N}\right)$ performs better than the conventional normal regression model $\left(R_{N} C_{N}\right)$, whereas the SE and RMSE are similar for the two models when the data are simulated from $R_{N} C_{N}$. Similarly, for $R_{T} C_{N}$ data, the coverage probability of the $95 \% \mathrm{BCl}$ of the conventional normal regression model $\left(R_{N} C_{N}\right)$ is higher than the nominal value (the $95 \% \mathrm{BCls}$ are conservative), whereas the robust regression model $\left(R_{T} C_{N}\right)$ yields coverage probabilities that are closer to the nominal value. For $R_{T} C_{N}$ data, the average length of the $95 \% \mathrm{BCls}$ from the conventional normal regression model $\left(R_{N} C_{N}\right)$ is approximately $16 \%$ larger than that of $95 \% \mathrm{BCls}$ from the robust regression model $\left(\mathrm{R}_{\mathrm{T}} \mathrm{C}_{\mathrm{N}}\right)$. For $R_{N} C_{N}$ data, the coverage probability of the $95 \%$ BCls from both models $\left(R_{N} C_{N}\right.$ and $\left.R_{T} C_{N}\right)$ is higher than the nominal value.

In summary, the robust regression model is competitive with and performs similarly to its 
non-robust counterpart when the TTP data come from $R_{N} C_{N}$, but the robust regression model performs better than its non-robust counterpart when the TTP data come from $\mathrm{R}_{T} \mathrm{C}_{\mathrm{N}}$.

\section{DISCUSSION}

Phase 2a and Phase 2b TB trials are conducted to respectively characterize the EBA and extended bactericidal activity of anti-TB drugs, before treatments enter the pivotal stages of drug development (i.e. Phase 3 trials to demonstrate long-term efficacy). EBA calculated from TTP data has recently become the preferred biomarker for the efficacy of such drugs.

The Bayesian NLME regression model of Burger and Schall ${ }^{[11]}$, which assumes normally distributed random coefficients and residuals, lacks robustness to gross outliers that often are present in the data. In this paper we fitted the robust regression models of Burger and Schall ${ }^{[17]}$ with different specifications of distributions for the residuals and random coefficients to a wide range of TTP datasets, spanning both 14-day EBA trials and 56-day extended bactericidal activity trials. When the performance of those regression models was assessed through model comparison statistics the DIC statistics and CLMMLs preferred the robust versions of the regression models in all cases. The estimates of the degrees of freedom and skewness parameters showed that the distribution of the model residuals is in most cases heavy tailed, and slightly skewed to the right. In all cases the robust models produced satisfactory fits to the data profiles. However, the datasets suggest that the skewness in the residuals, and magnitude of outliers in the vectors of random intercepts and slopes, are negligible. The specification of (symmetric) Student t distributions for residuals, and multivariate normal distributions for vectors of random intercepts and slopes, therefore seems adequate.

We conclude, therefore, that fitting a robust model to TTP data obviates the need for explicit identification and "deletion" of outliers, but nevertheless ensures that gross outliers exert no undue influence on the model fit. Furthermore, doing so considerably shortens and simplifies the blind data review before analysis starts. Thus we consider robust analysis as proposed here the preferred alternative to the explicit identification and removal of outliers from 
analyses. Furthermore, we found that $95 \%$ BCls for EBA were generally somewhat narrower for the robust models than for their non-robust counterparts, so that the robust methodology yields some gain in statistical power.

Given the importance of TTP as a surrogate marker for the efficacy TB treatments, robust versions of the Bayesian NLME regression model for TTP data (in particular, regression model $\mathrm{R}_{T} \mathrm{C}_{\mathrm{N}}$ ) should be fitted. Furthermore, the simulation study showed that heavy tailed distributions can better accommodate outliers in TTP data than the conventional normal distribution, resulting in more accurate EBA estimates, and credibility interval coverage closer to the nominal value. For future TB research, we recommend that the current practice of fitting conventional normal theory models (after exclusion of outliers) is abandoned in favor of fitting robust models to TTP data.

\section{APPENDIX}

The corresponding distributions specified for residuals and random coefficients (see Table 1) are discussed below in detail.

\section{Regression Model $1\left(\mathrm{R}_{\mathrm{N}} \mathrm{C}_{\mathrm{N}}\right)$ : Residuals: Normal}

\section{Random Coefficients: Multivariate Normal}

The regression model incorporates the assumption that the residuals follow i.i.d. normal distributions, i.e.:

$$
\varepsilon_{i j k} \mid \sigma_{\varepsilon_{j}}^{2} \sim N\left(0, \sigma_{\varepsilon_{j}}^{2}\right)
$$

where 0 and $\sigma_{\varepsilon j}^{2}$ are the mean and residual variances, respectively. The residual variances $\sigma_{\varepsilon j}^{2}$ are assigned inverse gamma prior distributions, namely:

$$
\sigma_{\varepsilon j}^{2} \sim I G(0.0001,0.0001)
$$


The random coefficients $\mu_{i j}$ were assumed to follow tri-variate normal distributions, and truncated normal distributions were specified for $\kappa_{i j}$ and $\gamma_{i j}$, namely:

$$
\begin{aligned}
\boldsymbol{\mu}_{i j} & \sim N\left(\boldsymbol{\mu}_{j}, \Omega_{\boldsymbol{\mu}_{j}}\right) \\
\kappa_{i j} & \sim T N\left(\kappa_{j}, \sigma_{\kappa_{j}}^{2}\right) I\left(2 \leq \kappa_{i j} \leq 11\right) \\
\gamma_{i j} & \sim T N\left(\gamma_{j}, \sigma_{\gamma_{j}}^{2}\right) I\left(0.1 \leq \gamma_{i j} \leq 2\right)
\end{aligned}
$$

where $\Omega_{\boldsymbol{\mu} j}, \sigma_{\kappa_{j}}^{2}$ and $\sigma_{\gamma_{j}}^{2}$ are respectively the covariance matrices of $\boldsymbol{\mu}_{\boldsymbol{i j}}$, and scale parameters of $\kappa_{i j}$ and $\gamma_{i j}$. Here, $I(x)$ denotes an indicator function taking the value 1 if $x$ is true, and 0 otherwise. Tri-variate normal and Wishart prior distributions are specified for $\boldsymbol{\mu}_{j}$ and $\Omega_{\boldsymbol{\mu}}^{-1}$, namely:

$$
\begin{aligned}
\boldsymbol{\mu}_{\boldsymbol{j}} & \sim N_{3}\left(\mathbf{0}, 10^{4} \times I_{3}\right) \\
\Omega_{\boldsymbol{\mu} j}^{-1} & \sim W\left(3,3 \times R_{j}\right)
\end{aligned}
$$

where $\mathbf{0}=(0,0,0)^{\prime}$ and $I_{3}$ denotes the $3 \times 3$ identity matrix. $R_{j}$ represent $3 \times 3$ inverse scale matrices. The methods by Kass and Natarajan ${ }^{[28]}$ were used for the choice of $R_{j}$. The parameters $\kappa_{j}, \gamma_{j}, \sigma_{\kappa_{j}}^{2}$ and $\sigma_{\gamma_{j}}^{2}$ are assumed to follow uniform prior distributions, namely:

$$
\begin{aligned}
\kappa_{j} & \sim U(2,11) \\
\gamma_{j} & \sim U(0.1,2) \\
\sigma_{\kappa_{j}}^{2} & \sim U(0.01,30) \\
\sigma_{\gamma_{j}}^{2} & \sim U(0.01,5)
\end{aligned}
$$

The bounds for $\kappa_{j}$ and $\gamma_{j}$ were chosen in such a way to avoid overfit of the first few and last few observations within the 14-day period of an EBA trial, and to allow for smooth transition between a few successive data points. ${ }^{[11]}$ 


\section{Regression Model $2\left(\mathrm{R}_{\mathrm{T}} \mathrm{C}_{\mathrm{N}}\right)$ : Residuals: Student $\mathrm{t}$}

\section{Random Coefficients: Multivariate Normal}

The regression model incorporates the assumption that the residuals follow i.i.d. Student $\mathrm{t}$ distributions, i.e.:

$$
\varepsilon_{i j k} \mid \sigma_{\varepsilon_{j}}^{2}, v_{j} \sim T\left(0, \sigma_{\varepsilon_{j}}^{2}, v_{j}\right)
$$

where $0, \sigma_{\varepsilon j}^{2}$ and $v_{j}$ are the mean, scale parameters and degrees of freedom, respectively. The degrees of freedom $v_{j}$ are assigned uniform prior distributions, namely:

$$
v_{j} \sim U(2,100)
$$

The prior distributions are specified similarly to those of regression model $R_{N} C_{N}$.

The supplementary material provides OpenBUGS code for the implementation of regression model $\mathrm{R}_{\mathrm{T}} \mathrm{C}_{\mathrm{N}}$ for a conventional 14-day EBA study.

\section{Regression Model $3\left(\mathrm{R}_{\mathrm{ST}} \mathrm{C}_{\mathrm{N}}\right)$ : Residuals: Skew Student $\mathrm{t}$}

\section{Random Coefficients: Multivariate Normal}

The regression model incorporates the assumption that the residuals follow i.i.d. skew Student $t$ distributions, ${ }^{[29]}$ i.e.:

$$
\varepsilon_{i j k} \mid \sigma_{\varepsilon_{j}}^{2}, \delta_{j}, v_{j} \sim S T\left(0, \sigma_{\varepsilon_{j}}^{2}, \delta_{j}, v_{j}\right)
$$

where $0, \sigma_{\varepsilon j}^{2}, \delta_{j}$ and $v_{j}$ are the mean, scale and skewness parameters, and degrees of freedom, respectively. The skew Student $t$ distribution is specified as a mixture of normal, truncated normal and gamma distributions (see Sahu et al. ${ }^{[29]}$ ). The skewness parameters $\delta_{j}$ are assigned normal prior distributions, namely:

$$
\delta_{j} \sim N\left(0,10^{4}\right)
$$


The prior distributions are specified similarly to those of regression model $R_{T} C_{N}$.

\section{Regression Model $4\left(\mathbf{R}_{\mathrm{ST}} \mathrm{C}_{\mathrm{T}}\right)$ : Residuals: Skew Student $\mathrm{t}$}

\section{Random Coefficients: Multivariate Student $t$}

The regression model incorporates the assumption that the vectors of random intercepts and slopes follow i.i.d. tri-variate Student t distributions, i.e.:

$$
\boldsymbol{\mu}_{i j} \mid \boldsymbol{\mu}_{j}, \Omega_{\boldsymbol{\mu}_{j}}, w_{j} \sim T_{3}\left(\boldsymbol{\mu}_{j}, \Omega_{\boldsymbol{\mu} j}, w_{j}\right)
$$

where $\boldsymbol{\mu}_{j}$ are the vectors of mean intercepts and slopes, and $\Omega_{\boldsymbol{\mu}}$ and $w_{j}$ are scale matrices and degrees of freedom, respectively. The degrees of freedom $w_{j}$ are assigned uniform prior distributions, namely:

$$
w_{j} \sim U(2,100)
$$

The prior distributions are specified similarly to those of regression model $R_{S T} C_{N}$.

\section{REFERENCES}

[1] Mitchison DA. Clinical development of anti-tuberculosis drugs. J. Antimicrob. Chemother. 2006; 58: 494-495.

[2] Svensson EM, Karlsson MO. Modelling of mycobacterial load reveals bedaquiline's exposure-response relationship in patients with drug-resistant TB. J. Antimicrob. Chemother. 2017; 72(12): 3398-3405.

[3] Phillips PPJ, Mendel CM, Burger DA, Crook AM, Nunn AJ, Dawson R, Diacon AH, Gillespie SH. Limited role of culture conversion for decision-making in individual patient care and for advancing novel regimens to confirmatory clinical trials. BMC Medicine 2016; 14: 19. DOI: $10.1186 / \mathrm{s} 12916-016-0565-\mathrm{y}$.

[4] Wallis RS, Doherty TM, Onyebujoh P, Vahedi M, Laang H, Olesen O, Parida S, Zumla A. 
Biomarkers for tuberculosis disease activity, cure, and relapse. The Lancet Infect. Dis. 2009; 9: 162-172.

[5] Weiner M, Prihoda TJ, Burman W, Johnson JL, Goldberg S, Padayatchi N, Duran P, Engle M, Muzanye G, Mugerwa RD, et al. Evaluation of time to detection of Mycobacterium tuberculosis in broth culture as a determinant for end points in treatment trials. J. Clin. Microbiol. 2010; 48(12): 4370-4376.

[6] Dawson R, Diacon AH, Everitt D, Van Niekerk C, Donald PR, Burger DA, Schall R, Spigelman M, Conradie A, Eisenach K, et al. Efficiency and safety of the combination of moxifloxacin, pretomanid (PA-824), and pyrazinamide during the first 8 weeks of antituberculosis treatment: A phase $2 \mathrm{~b}$, open-label, partly randomised trial in patients with drug-susceptible or drug-resistant pulmonary tuberculosis. The Lancet 2015; 385(9979): 1738-1747. DOI: 10.1016/S0140-6736(14)62002-X.

[7] Bark CM, Gitta P, Ogwang S, Nsereko M, Thiel BA, Boom WH, Eisenach KD, Joloba ML, Johnson JL. Comparison of time to positive and colony counting in an early bactericidal activity study of anti-tuberculosis treatment. The Int. J. Tuberc. Lung Dis. 2013; 17(11): 1448-1451.

[8] Diacon AH, Maritz JS, Venter A, Van Helden PD, Dawson R, Donald PR. Time to liquid culture positivity as a substitute for colony counting on agar plates in early bactericidal activity studies of antituberculosis agents. Clin. Microbiol. Infect. 2012; 18(7): 711-717.

[9] Sloan DJ, Corbett EL, Butterworth AE, Mwandumba HC, Khoo SH, Mdolo A, Shani D, Kamdolozi M, Allen J, Mitchison DA, et al. Optimizing outpatient serial sputum colony counting for studies of tuberculosis treatment in resource-poor settings. J. Clin. Microbiol. 2012; 50(7): 2315-2320. DOI: 10.1128/JCM.00043-12.

[10] Diacon AH, Dawson R, Von Groote-Bidlingmaier F, Symons G, Venter A, Donald PR, Van Niekerk C, Everitt D, Hutchings J, Burger DA, et al. Bactericidal activity of pyrazi- 
namide and clofazimine alone and in combinations with pretomanid and bedaquiline. Am. J. Respir. Critical Care Medicine 2015; 191(8): 943-953. DOI: 10.1164/rccm.20141018010C.

[11] Burger DA, Schall R. A Bayesian nonlinear mixed-effects regression model for the characterization of early bactericidal activity of tuberculosis drugs. J. Biopharm. Stat. 2015; 25(6): 1247-1271. DOI: 10.1080/10543406.2014.971170.

[12] Lachos VH, Castro LM, Dey DK. Bayesian inference in nonlinear mixed-effects models using normal independent distributions. Comput. Stat. \& Data Analysis 2013; 64: 237252.

[13] Wang C, Blei DM. A general method for robust Bayesian modeling. Bayesian Analysis 2018; TBA: DOI: 10.1214/17-BA1090.

[14] De la Cruz R. Bayesian analysis for nonlinear mixed-effects models under heavy-tailed distributions. Pharm. Stat. 2014; 13(1): 81-93.

[15] Diacon AH, Dawson R, Von Groote-Bidlingmaier F, Symons G, Venter A, Donald PR, Van Niekerk C, Everitt D, Winter H, Becker P, et al. 14-day bactericidal activity of PA824, bedaquiline, pyrazinamide, and moxifloxacin combinations: A randomized trial. The Lancet 2012; 380(9846): 986-993. DOI: 10.1016/S0140-6736(12)61080-0.

[16] ICH. ICH E9: Statistical Principles for Clinical Trials. 1998.

[17] Burger DA, Schall R. Robust fit of Bayesian mixed effects regression models with application to colony forming unit count in tuberculosis research. Stat. Medicine 2018; 37(4): 544-556.

[18] Kassir N. Quantitative analysis of MGIT time to positivity using a two-part longitudinal model in patients with pulmonary tuberculosis: A meta-analysis of 11 clinical studies in- 
cluding 30 unique regimens. In: $10^{\text {th }}$ International Workshop on Clinical Pharmacology of Tuberculosis Drugs. 2017:1-22.

[19] Chigutsa E, Patel K, Denti P, Visser M, Maartens G, Kirkpatrick CMJ, Mcllleron H, Karlsson MO. A time-to-event pharmacodynamic model describing treatment response in patients with pulmonary tuberculosis using days to positivity in automated liquid mycobacterial culture. Antimicrob. Agents Chemother. 2013; 57(2): 789-795.

[20] Lunn DJ, Spiegelhalter DJ, Thomas A, Best NG. The BUGS project: Evolution, critique and future directions. Stat. Medicine 2009; 28(25): 3049-3067. DOI: 10.1002/sim.3680.

[21] Ntzoufras I. Bayesian Modeling Using WinBUGS. Hoboken, New Jersey: John Wiley \& Sons, Inc.; 2009.

[22] Brooks SP, Gelman A. General methods for monitoring convergence of iterative simulations. J. Comput. Graph. Stat. 1998; 7(4): 434-455. DOI: 10.1080/10618600.1998.10474787.

[23] Hahn H, Bouvier A, Kiêu K. R2Cuba: Multidimensional numerical integration; 2013. R package Version 1.0-11; URL http: //CRAN . R-project. org/package=R2Cuba.

[24] Diacon AH, Dawson R, Von Groote-Bidlingmaier F, Symons G, Venter A, Donald PR, Conradie A, Erondu N, Ginsberg AM, Egizi E, et al. Randomized dose-ranging study of the 14-day early bactericidal activity of bedaquiline (TMC207) in patients with sputum microscopy smear-positive pulmonary tuberculosis. Antimicrob. Agents Chemother. 2013; 57(5): 2199-2203. DOI: 10.1128/AAC.02243-12.

[25] Diacon AH, Dawson R, Hanekom M, Narunsky K, Maritz SJ, Venter A, Donald PR, Van Niekerk C, Whitney K, Rouse DJ, et al. Early bactericidal activity and pharmacokinetics of PA-824 in smear-positive tuberculosis patients. Antimicrob. Agents Chemother. 2010; 54(8): 3402-3407. DOI: 10.1128/AAC.01354-09. 
[26] Diacon AH, Dawson R, Du Bois J, Narunsky K, Venter A, Donald PR, Van Niekerk C, Erondu N, Ginsberg AM, Becker P, et al. Phase II dose-ranging trial of the early bactericidal activity of PA-824. Antimicrob. Agents Chemother. 2012; 56(6): 3027-3031. DOI: 10.1128/AAC.06125-11.

[27] Juárez MA, Steel MFJ. Model-based clustering of non-Gaussian panel data based on skew-t distributions. J. Bus. \& Econ. Stat. 2010; 28(1): 52-66.

[28] Kass RE, Natarajan R. A default conjugate prior for variance components in generalized linear mixed models (comments on article by Browne and Draper). Bayesian Analysis 2006; 1(3): 535-542.

[29] Sahu SK, Dey DK, Branco MD. A new class of multivariate skew distributions with applications to Bayesian regression models. The Can. J. Stat. 2003; 31(2): 129-150. DOI: $10.2307 / 3316064$.

Table 1: Model Specifications for Bayesian Mixed Effects Regression Models

\begin{tabular}{llll}
\hline & & \multicolumn{2}{c}{ Distributions } \\
\cline { 3 - 4 } Regression Model & No. & Residuals & Random Coefficients \\
\hline $\mathrm{R}_{\mathrm{N}} \mathrm{C}_{\mathrm{N}}$ & 1 & Normal & Multivariate normal \\
$\mathrm{R}_{\mathrm{T}} \mathrm{C}_{\mathrm{N}}$ & 2 & Student $\mathrm{t}$ & Multivariate normal \\
$\mathrm{R}_{\mathrm{ST}} \mathrm{C}_{\mathrm{N}}$ & 3 & Skew Student $\mathrm{t}$ & Multivariate normal \\
$\mathrm{R}_{\mathrm{ST}} \mathrm{C}_{\mathrm{T}}$ & 4 & Skew Student $\mathrm{t}$ & Multivariate Student $\mathrm{t}$ \\
\hline
\end{tabular}


Table 2: Comparison of Bayesian NLME Regression Models Using DIC Statistics and CLMMLs

\begin{tabular}{llrrrr}
\hline & & \multicolumn{4}{c}{ Regression Model } \\
\cline { 3 - 6 } Trial & Statistic & $\mathbf{R}_{\mathbf{N}} \mathbf{C}_{\mathbf{N}}$ & $\mathbf{R}_{\mathrm{T}} \mathbf{C}_{\mathbf{N}}$ & $\mathbf{R}_{\mathbf{S T}} \mathbf{C}_{\mathbf{N}}$ & $\mathbf{R}_{\mathbf{S T}} \mathbf{C}_{\mathbf{T}}$ \\
\hline CL001 & DIC & $-2022^{\{4\}}$ & $-2249^{\{1,2\}}$ & $-2247^{\{3\}}$ & $-2249^{\{1,2\}}$ \\
& CLMML & $386.36^{\{4\}}$ & $456.09^{\{1\}}$ & $410.01^{\{3\}}$ & $417.45^{\{2\}}$ \\
CL007 & DIC & $-1335^{\{4\}}$ & $-1629^{\{3\}}$ & $-1636^{\{2\}}$ & $-1638^{\{1\}}$ \\
& CLMML & $86.29^{\{4\}}$ & $198.62^{\{1\}}$ & $164.56^{\{3\}}$ & $166.27^{\{2\}}$ \\
CL010 & DIC & $-1933^{\{4\}}$ & $-2174^{\{3\}}$ & $-2189^{\{1,2\}}$ & $-2189^{\{1,2\}}$ \\
NC001 & CLMML & $285.98^{\{4\}}$ & $427.86^{\{1\}}$ & $371.15^{\{3\}}$ & $375.87^{\{2\}}$ \\
& DIC & $-3303^{\{4\}}$ & $-3768^{\{1\}}$ & $-3767^{\{2\}}$ & $-3764^{\{3\}}$ \\
NC002 & CLMML & $874.11^{\{4\}}$ & $1022.18^{\{1\}}$ & $970.83^{\{3\}}$ & $973.46^{\{2\}}$ \\
& DIC & $-752^{\{4\}}$ & $-1038^{\{2\}}$ & $-1047^{\{1\}}$ & $-1037^{\{3\}}$ \\
NC003 & CLMML & $-54.65^{\{4\}}$ & $22.61^{\{1\}}$ & $-6.29^{\{3\}}$ & $-3.13^{\{2\}}$ \\
& DIC & $N E$ & $-4653^{\{3\}}$ & $-4676^{\{1\}}$ & $-4677^{\{2\}}$ \\
& CLMML & $837.14^{\{4\}}$ & $1239.65^{\{1\}}$ & $1211.02^{\{2\}}$ & $1203.87^{\{3\}}$ \\
\hline
\end{tabular}

Note: $\mathrm{R}_{\mathrm{N}} \mathrm{C}_{\mathrm{N}}$ : Residuals and random coefficients assumed to follow normal distributions. $\mathrm{R}_{\mathrm{T}} \mathrm{C}_{\mathrm{N}}$ : Residuals and random coefficients respectively assumed to follow Student $t$ and trivariate normal distributions. $\mathrm{R}_{S T} \mathrm{C}_{\mathrm{N}}$ : Residuals and random coefficients respectively assumed to follow skew Student $t$ and tri-variate normal distributions. $\mathrm{R}_{S_{T}} \mathrm{C}_{\mathrm{T}}$ : Residuals and random coefficients respectively assumed to follow skew Student $t$ and tri-variate Student $t$ distributions. DIC: Deviance information criterion. CLMML: Compound Laplace-Metropolis marginal likelihood on the logarithmic scale. NE: Not estimable. NLME: Nonlinear mixed effects. Superscripts indicate the ranking of model comparison statistics from most favored to least favored. 
Table 3: Posterior Estimates and $95 \%$ BCls of Mean $E_{B A} A_{T P_{j}}(0-56): R_{N} C_{N}$ and $R_{T} C_{N}-$ NC002 Trial

\begin{tabular}{|c|c|c|c|c|}
\hline \multirow[b]{3}{*}{ Treatment Group } & \multicolumn{4}{|c|}{ Regression Model } \\
\hline & \multicolumn{2}{|c|}{$\mathrm{R}_{\mathrm{N}} \mathrm{C}_{\mathrm{N}}$} & \multicolumn{2}{|c|}{$\mathrm{R}_{\mathrm{T}} \mathrm{C}_{\mathrm{N}}$} \\
\hline & Point Estimate & $95 \% \mathrm{BCl}$ & Point Estimate & $95 \% \mathrm{BCl}$ \\
\hline M-PA100-Z & 1.840 & {$[1.506 ; 2.163]$} & 2.012 & {$[1.755 ; 2.274]$} \\
\hline M-PA200-Z & 1.918 & {$[1.670 ; 2.159]$} & 2.007 & {$[1.813 ; 2.201]$} \\
\hline M-PA200-Z-MDR & 1.572 & {$[0.809 ; 2.240]$} & 1.683 & {$[0.872 ; 2.408]$} \\
\hline Rifafour & 1.582 & {$[1.341 ; 1.822]$} & 1.712 & {$[1.508 ; 1.908]$} \\
\hline
\end{tabular}

Note: $\mathrm{R}_{\mathrm{N}} \mathrm{C}_{\mathrm{N}}$ : Residuals and random coefficients assumed to follow normal distributions. $\mathrm{R}_{\mathrm{T}} \mathrm{C}_{\mathrm{N}}$ : Residuals and random coefficients respectively assumed to follow Student $\mathrm{t}$ and trivariate normal distributions. BCl: Bayesian credibility interval. $\operatorname{EBA}_{\operatorname{TTP}_{j}}\left(t_{1}-t_{2}\right)$ : Daily percentage change in TTP from Day $t_{1}$ to Day $t_{2}$ of treatment group $j$. TTP: Time to positivity. 
Table 4: Simulation Study of Bayesian NLME Regression Models: Accuracy and Precision of $\mathrm{EBA}_{\mathrm{TTP}_{j}}(0-14)$ Estimates, and Coverage of $95 \% \mathrm{BCls}$

\begin{tabular}{|c|c|c|c|c|c|}
\hline \multirow[b]{3}{*}{$\mathrm{EBA}_{\mathrm{TTP}_{j}}(0-14)$} & \multirow[b]{3}{*}{ Statistic } & \multicolumn{4}{|c|}{ Regression Model } \\
\hline & & \multicolumn{2}{|c|}{ Simulated From $\mathbf{R}_{N} C_{N}$} & \multicolumn{2}{|c|}{ Simulated From $\mathbf{R}_{T} \mathbf{C}_{N}$} \\
\hline & & $\mathbf{R}_{\mathrm{N}} \mathbf{C}_{\mathrm{N}}$ & $\mathbf{R}_{\mathrm{T}} \mathbf{C}_{\mathrm{N}}$ & $\mathrm{R}_{\mathrm{N}} \mathrm{C}_{\mathrm{N}}$ & $\mathbf{R}_{\mathrm{T}} \mathbf{C}_{\mathrm{N}}$ \\
\hline \multirow[t]{4}{*}{1.0989} & Bias & 0.11 & 0.14 & -0.03 & -0.09 \\
\hline & SE & 2.7552 & 2.7298 & 3.3067 & 2.8409 \\
\hline & RMSE & 2.7563 & 2.7324 & 3.3058 & 2.8415 \\
\hline & $95 \% \mathrm{BCl}$ coverage (\%) & 96.67 & 97.53 & 97.07 & 95.80 \\
\hline \multirow[t]{4}{*}{4.0008} & Bias & 0.01 & 0.04 & 0.19 & 0.15 \\
\hline & SE & 2.7891 & 2.8028 & 3.7035 & 2.8701 \\
\hline & RMSE & 2.7882 & 2.8022 & 3.7070 & 2.8733 \\
\hline & $95 \% \mathrm{BCl}$ coverage (\%) & 97.20 & 96.60 & 96.93 & 95.93 \\
\hline
\end{tabular}

Note: $\mathrm{R}_{\mathrm{N}} \mathrm{C}_{\mathrm{N}}$ : Residuals and random coefficients assumed to follow normal distributions. $\mathrm{R}_{T} \mathrm{C}_{\mathrm{N}}$ : Residuals and random coefficients respectively assumed to follow Student $\mathrm{t}$ and trivariate normal distributions. BCl: Bayesian credibility interval. $\operatorname{EBA}_{\operatorname{TTP}_{j}}\left(t_{1}-t_{2}\right)$ : Daily percentage change in TTP from Day $t_{1}$ to Day $t_{2}$ of treatment group $j$. NLME: Nonlinear mixed effects. RMSE: Root mean square error. SE: Standard error. TTP: Time to positivity. 
Figure 1: Observed and Fitted $\log _{10}(T T P)$ vs. Time Profiles Containing No Outliers: $R_{N} C_{N}$ and $\mathrm{R}_{\mathrm{ST}} \mathrm{C}_{\mathrm{T}}-\mathrm{NC003}$ Trial

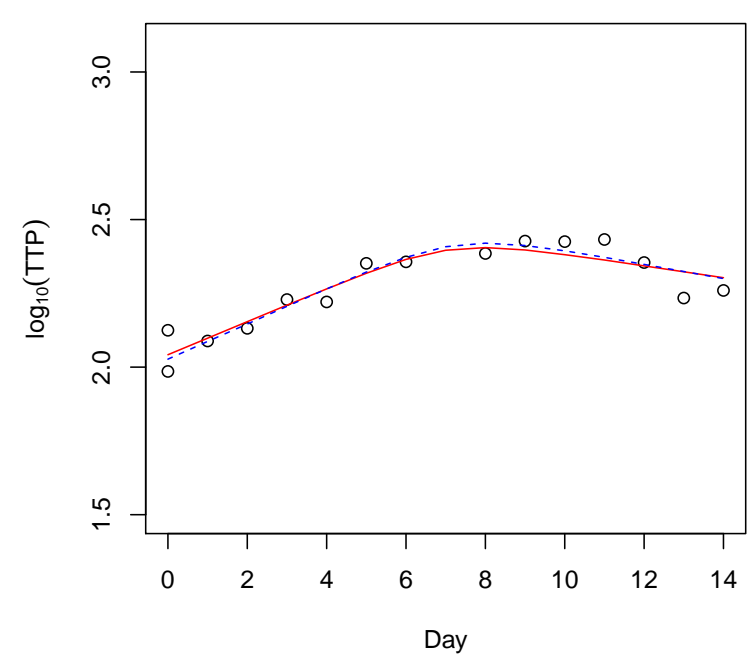

- Observed $\Delta$ Censored

Model
$-\mathrm{R}_{\mathrm{N}} \mathrm{C}_{\mathrm{N}} \ldots \mathrm{R}_{\mathrm{ST}} \mathrm{C}_{\mathrm{T}}$



- Observed $\Delta$ Censored

Model
$-\mathrm{R}_{\mathrm{N}} \mathrm{C}_{\mathrm{N}} \ldots \mathrm{R}_{\mathrm{ST}} \mathrm{C}_{\mathrm{T}}$

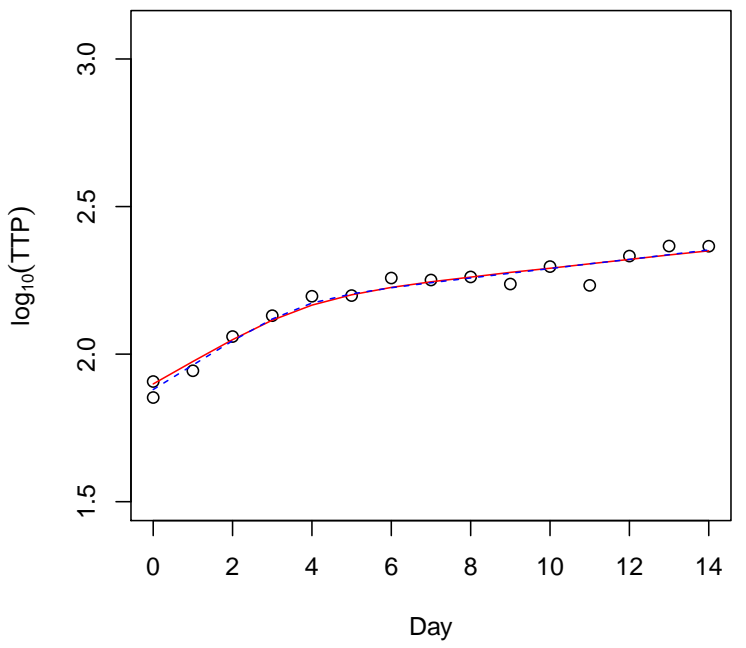

- Observed $\Delta$ Censored

Model

$-\mathrm{R}_{\mathrm{N}} \mathrm{C}_{\mathrm{N}} \ldots \mathrm{R}_{\mathrm{ST}} \mathrm{C}_{\mathrm{T}}$

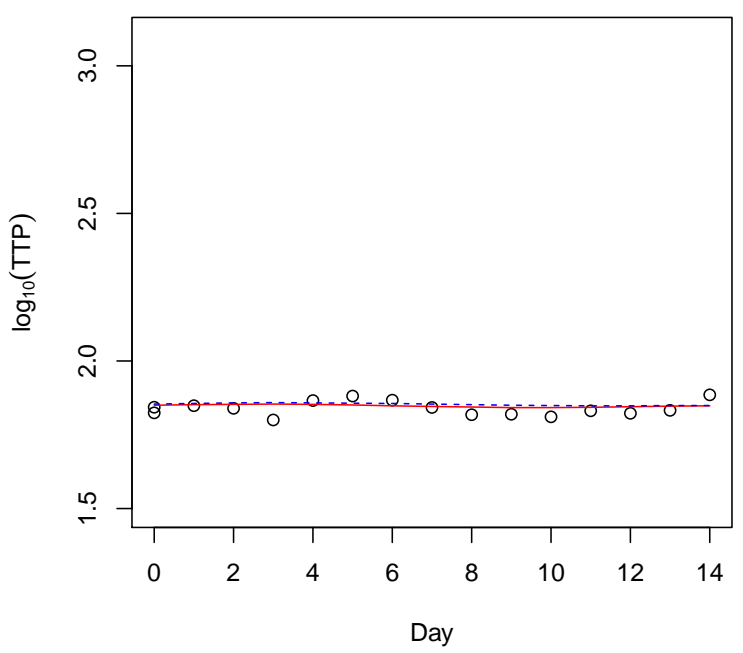

- Observed $\Delta$ Censored

Model

$-\mathrm{R}_{\mathrm{N}} \mathrm{C}_{\mathrm{N}} \ldots \mathrm{R}_{\mathrm{ST}} \mathrm{C}_{\mathrm{T}}$

Note: $R_{N} C_{N}$ : Residuals and random coefficients assumed to follow normal distributions. $\mathrm{R}_{S T} \mathrm{C}_{\mathrm{T}}$ : Residuals and random coefficients respectively assumed to follow skew Student $t$ and tri-variate Student t distributions. TTP: Time to positivity. Censored: TTP specified as rightcensored values on the logarithmic scale to the base of 10 . 
Figure 2: Observed and Fitted $\log _{10}\left(\right.$ TTP) vs. Time Profiles Containing Gross Outliers: $R_{N} C_{N}$ and $\mathrm{R}_{\mathrm{ST}} \mathrm{C}_{\mathrm{T}}-\mathrm{NC003}$ Trial

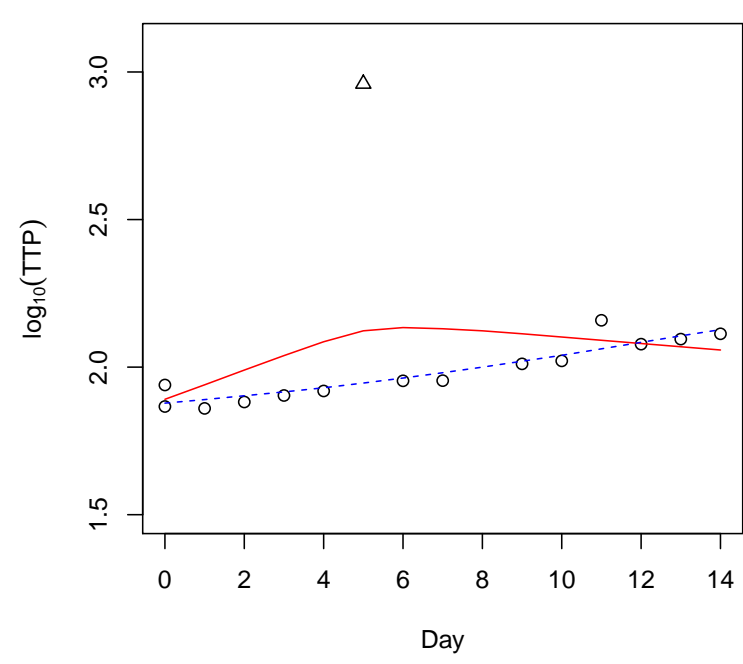

- Observed $\Delta$ Censored
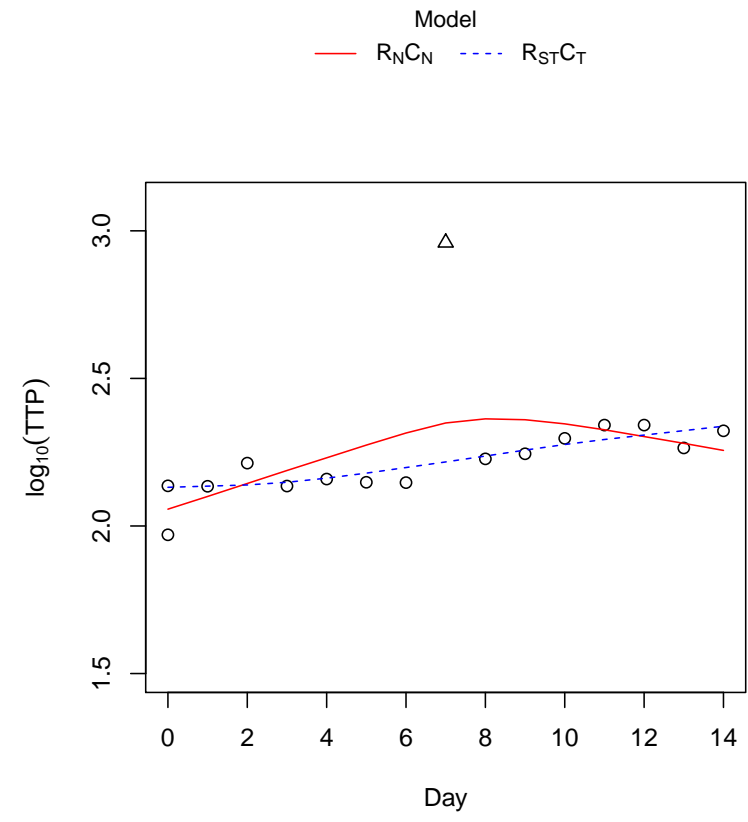

- Observed $\Delta$ Censored

Model

$-\mathrm{R}_{\mathrm{N}} \mathrm{C}_{\mathrm{N}} \ldots \mathrm{R}_{\mathrm{ST}} \mathrm{C}_{\mathrm{T}}$

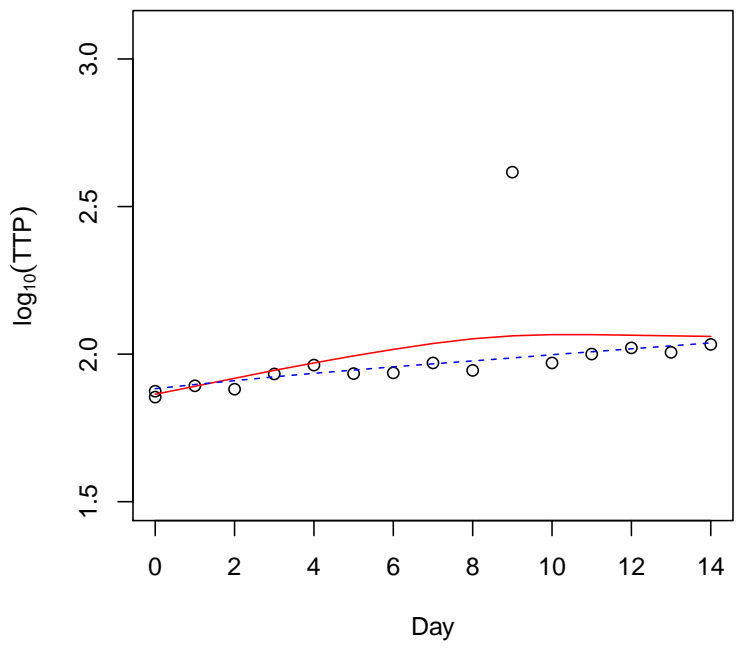

- Observed $\Delta$ Censored

Model

$\longrightarrow \mathrm{R}_{\mathrm{N}} \mathrm{C}_{\mathrm{N}} \ldots \mathrm{R}_{\mathrm{ST}} \mathrm{C}_{\mathrm{T}}$

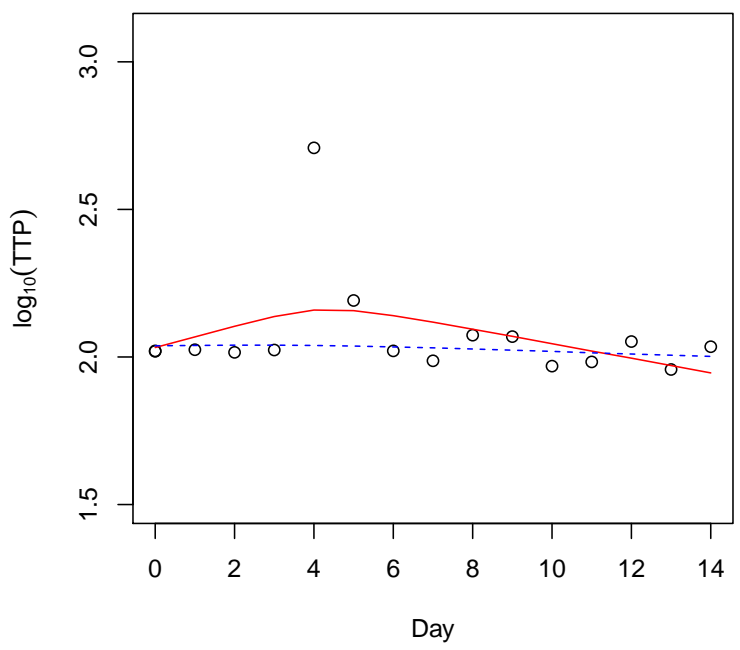

- Observed $\Delta$ Censored Model

$\longrightarrow \mathrm{R}_{\mathrm{N}} \mathrm{C}_{\mathrm{N}} \ldots \mathrm{R}_{\mathrm{ST}} \mathrm{C}_{\mathrm{T}}$

Note: $\mathrm{R}_{N} \mathrm{C}_{\mathrm{N}}$ : Residuals and random coefficients assumed to follow normal distributions. $\mathrm{R}_{S T} \mathrm{C}_{\mathrm{T}}$ : Residuals and random coefficients respectively assumed to follow skew Student $t$ and tri-variate Student t distributions. TTP: Time to positivity. Censored: TTP specified as rightcensored values on the logarithmic scale to the base of 10 . 
Figure 3: Bland-Altman Plot of Posterior Estimates of Mean $E_{B A A_{j}}(0-14): R_{N} C_{N} v s . R_{S T} C_{T}$



Trial $\mathrm{OCL001} \triangle \mathrm{CL007}+\mathrm{CL} 010 \times \mathrm{NC001} \diamond \mathrm{NC002} \nabla \mathrm{NC003}$

Note: Differences between $R_{N} C_{N}$ versus $R_{S T} C_{T}$ in the posterior estimates of mean $\mathrm{EBA}_{\mathrm{TTP}_{j}}(0-14)$ plotted against the corresponding averages (32 treatment groups). Horizontal lines denote the point estimate and $95 \% \mathrm{Cl}$ of associated differences. $\mathrm{R}_{\mathrm{N}} \mathrm{C}_{\mathrm{N}}$ : Residuals and random coefficients assumed to follow normal distributions. $\mathrm{R}_{\mathrm{ST}} \mathrm{C}_{\mathrm{T}}$ : Residuals and random coefficients respectively assumed to follow skew Student $t$ and tri-variate Student $t$ distributions. Cl: Confidence interval. EBA TTP $_{j}\left(t_{1}-t_{2}\right)$ : Daily percentage change in TTP from Day $t_{1}$ to Day $t_{2}$ of treatment group $j$. TTP: Time to positivity. 
Figure 4: Ratio of Width of $95 \% \mathrm{BCl}$ for Mean $\operatorname{EBA}_{T T P_{j}}(0-14): \mathrm{R}_{\mathrm{T}} \mathrm{C}_{\mathrm{N}}, \mathrm{R}_{\mathrm{ST}_{\mathrm{T}}} \mathrm{C}_{\mathrm{N}}$ and $\mathrm{R}_{\mathrm{ST}_{\mathrm{T}}} \mathrm{C}_{\mathrm{T}}$ vs. $\mathrm{R}_{\mathrm{N}} \mathrm{C}_{\mathrm{N}}$

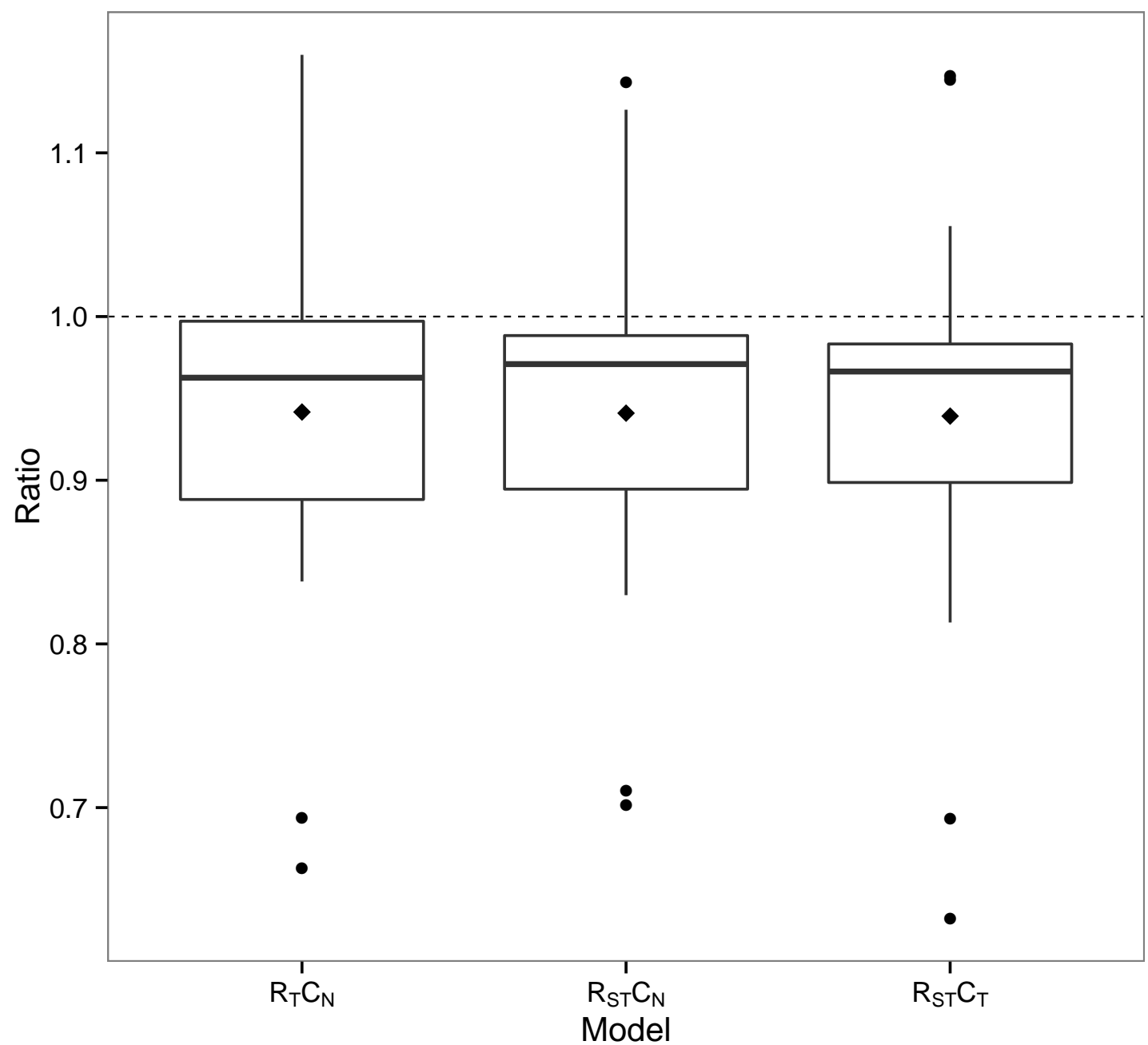

Note: Shown are ratios from 32 treatment groups. $R_{N} C_{N}$ : Residuals and random coefficients assumed to follow normal distributions. $\mathrm{R}_{T} \mathrm{C}_{\mathrm{N}}$ : Residuals and random coefficients respectively assumed to follow Student $t$ and tri-variate normal distributions. $\mathrm{R}_{\mathrm{ST}} \mathrm{C}_{\mathrm{N}}$ : Residuals and random coefficients respectively assumed to follow skew Student $t$ and tri-variate normal distributions. $\mathrm{R}_{\mathrm{ST}} \mathrm{C}_{\mathrm{T}}$ : Residuals and random coefficients respectively assumed to follow skew Student $t$ and tri-variate Student $t$ distributions. BCl: Bayesian credibility interval. $\mathrm{EBA}_{\mathrm{TTP}_{j}}\left(t_{1}-t_{2}\right)$ : Daily percentage change in TTP from Day $t_{1}$ to Day $t_{2}$ of treatment group $j$. TTP: Time to positivity. 
Figure 5: Posterior Estimates of Residual Degrees of Freedom and Skewness Parameters: $\mathrm{R}_{S T} \mathrm{C}_{\mathrm{T}}$



(a) $v_{j}$



(b) $\delta_{j}$

Note: Shown are estimates from 32 treatment groups. $\mathrm{R}_{\mathrm{ST}} \mathrm{C}_{\mathrm{T}}$ : Residuals and random coefficients respectively assumed to follow skew Student $t$ and tri-variate Student $t$ distributions. 
Figure 6: Posterior Estimates and $95 \%$ BCls of Mean $\log _{10}(T T P): R_{N} C_{N}$ and $R_{T} C_{N}-N C 002$ Trial



(a) M-PA100-Z

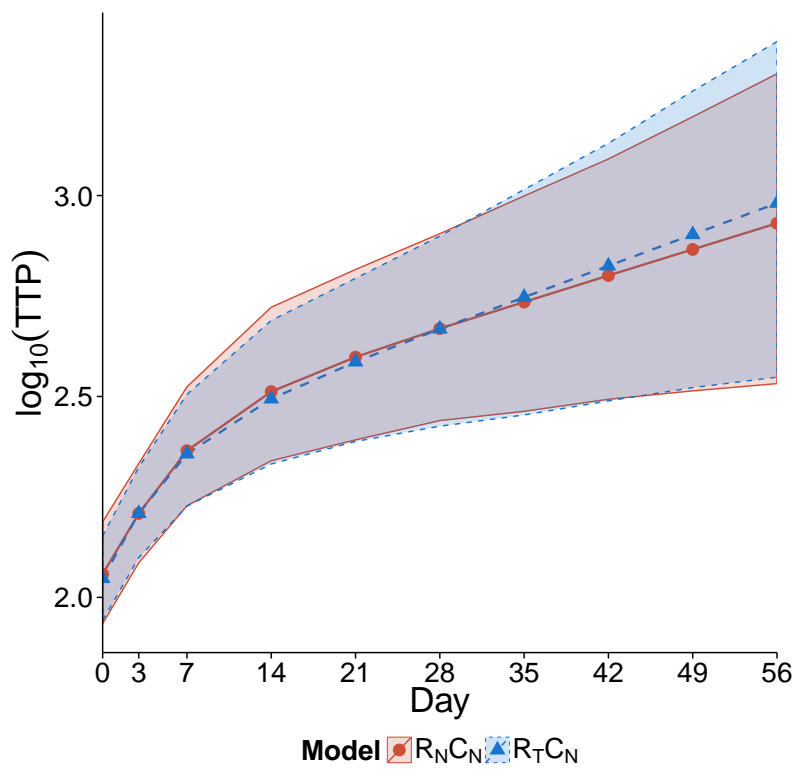

(c) M-PA200-Z-MDR

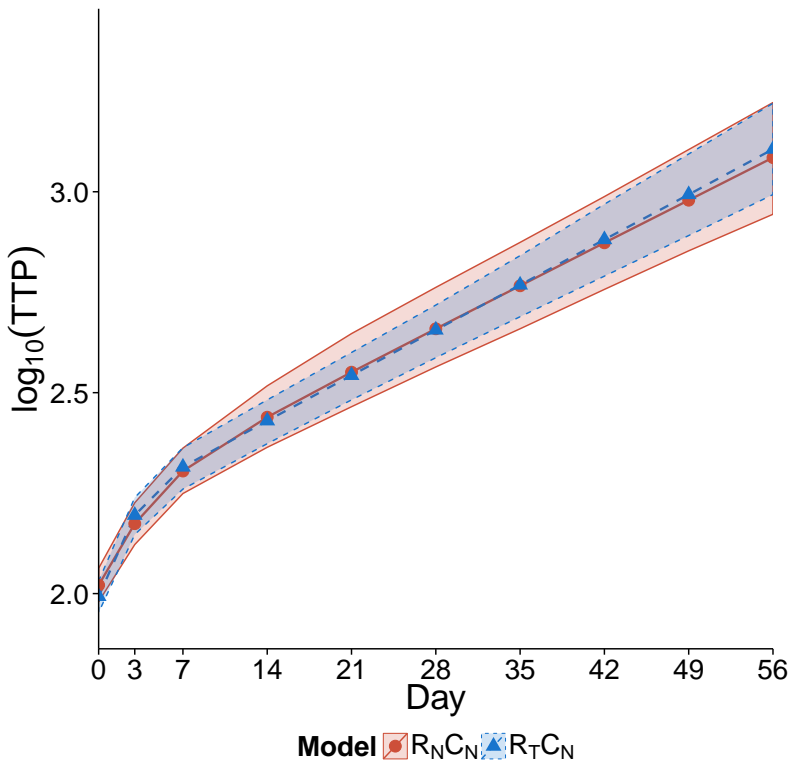

(b) M-PA200-Z



(d) Rifafour

Note: $\mathrm{R}_{\mathrm{N}} \mathrm{C}_{\mathrm{N}}$ : Residuals and random coefficients assumed to follow normal distributions. $\mathrm{R}_{\mathrm{T}} \mathrm{C}_{\mathrm{N}}$ : Residuals and random coefficients respectively assumed to follow Student $t$ and trivariate normal distributions. BCl: Bayesian credibility interval. TTP: Time to positivity. 